tionellen Medien ausbreiten und spätestens dann eine große Kraft entfalten können - und das Web so zu einem wichtigen Werkzeug für soziale Bewegungen werden kann.

In den Beiträgen des letzten Abschnittes werden einige Websites auf Potenziale für Partizipation und zivilgesellschaftliches Engagement hin untersucht. Anders Svensson argumentiert vor dem Hintergrund seiner Analyse von über 4000 Diskussionsbeiträgen im Online-Forum eines schwedischen Profi-Hockeyteams, dass schon die Diskurse in ganz politikfernen Räumen offenkundig eine Keimzelle von Bürgerschaftlichkeit und demokratischer Diskussionskultur seien. Schließlich konstituierten die User die Regeln und Normen der Diskussion selbst und trügen auch selbst dafür Sorge, dass diese in Geltung blieben. Ulf Buskqvists belegt in seinem Artikel mithilfe kritischer Diskursanalyse, dass sich die Partizipationsangebote auf Websites stark daran orientieren, welche Ziele und Strategien die Produzenten dieser Websites (hier: Parteien, Medien, zivilgesellschaftliche Akteure/Jugendorganisationen) verfolgen. Während die erstgenannten Akteure nur die "Illusion von Interaktivität“ erzeugen, neigen zivilgesellschaftliche Akteure eher dazu, die Programmkontrolle zu dezentralisieren und z. B. Foren-Threads nicht allzu restriktiv zu moderieren. Fredrik Miegel und Tobias Olsson weisen in einem der richtungsweisendsten Aufsätze des Bandes jedoch darauf hin, dass die Internetforschung nach wie vor an der Engführung leidet, dass meist nur entweder über die Produzenten oder über die Inhalte oder über die User geforscht wird. Sie fordern und nutzen daher einen Analyserahmen, der alle drei Phänomenbereiche berücksichtigt. Am Beispiel des Scheiterns einer mit dem Ziel der Mobilisierung der schwedischen Wählerschaft vor den Wahlen im Jahr 2006 gestarteten Website zeigen die Autoren auf, welche Erklärungskraft ein solcher dreigliedriger Ansatz entfalten kann.

Wird der Sammelband nun dem eigenen Anspruch gerecht, eine neue Nüchternheit in die sozial- und kommunikationswissenschaftliche Internet-Forschung einzubringen? Wie so oft bei diesem Format muss die Antwort lauten: teilweise! Leider krankt ein nicht zu vernachlässigender Teil der Beiträge an mangelnder inhaltlicher Passung, unklaren theoretischen Vorannahmen und methodischen Unschärfen. Oft wird die benutze Methode (meist qualitative Inhaltsanalyse oder kritische Diskursanalyse) keiner kritischen Reflexion unterzogen. Andere Beiträge aber - zuvörderst die von Ward, Duits/van Zoonen/Hirzalla, Aydemir/
Apak, Svensson und Miegel/Olsson - begeistern, indem sie frei von den genannten Schwächen und voller wertvoller Perspektiven einer Theorie der Bürgergesellschaft Dahlgrenscher Prägung sind, die einer aufgeklärten demokratietheoretischen Internetforschung gut tun werden.

Christoph Meißelbach

\section{Tina Rohowski}

\section{Das Private in der Politik}

Politiker-Homestories in der deutschen Unterhaltungspresse

Wiesbaden: VS Research, 2009-163 S.

\section{ISBN 978-3-531-16865-4}

„Kinkel wird Mensch: Außenminister Klaus Kinkel über Gefühle, Familie, Kohl, Haare färben und das Elend der Welt" („Kinkel wird Mensch“. In: Bunte 48, 39 (1995), S. 103ff.). Es ist diese - mitunter beinahe absurd anmutende - Mischung aus Banalität und politischer Bedeutsamkeit, die sog. Homestories auszeichnet. Am heimischen Wohnzimmertisch, in der Ferienwohnung oder sogar im Pool planschend gewähren Politiker einen Einblick in das, was wie ihr Privatleben daherkommt - und im besten Fall eine gute Inszenierung dessen ist, was die Rezipienten für den Politikeralltag halten sollen.

Kommunikations- und Politikwissenschaft haben diese etablierte Form der Politik(er)berichterstattung in der Vergangenheit geflissentlich ignoriert. Den Homestories wird das Schicksal zuteil, das viele unterhaltende Darstellungsformen ereilt - die Zahl der Rezipienten ist groß, die wissenschaftliche Ignoranz ist es auch. Tina Rohowski setzt mit ihrer Diplomarbeit dort an, wo sich andere Autoren bislang mit Pauschalurteilen, Vermutungen und Verurteilungen zufrieden gaben. Sie leistet mit ihrer Untersuchung einen wichtigen Beitrag zur politischen Kommunikationsforschung.

Tina Rohowski untersucht das Genre seit seinem Entstehen nach dem Zweiten Weltkrieg, nimmt sich der Frage an, wie Homestories in den deutschen Publikumszeitschriften Stern und Bunte über das Privatleben von Politikern berichten, wie sich die Berichterstattung im Laufe der letzten Jahrzehnte verändert hat und ob es, wie häufig postuliert, tatsächlich eine $\mathrm{Zu}-$ nahme solcher Inhalte gibt.

Auf das einleitende Kapitel folgt eine Analyse des Verhältnisses von Massenmedien und Politik. Tina Rohowski gelingt es, den umfang- 
reichen und diffusen Forschungsstand knapp zusammenzufassen. Das dritte Kapitel beleuchtet die Rolle des Privaten in der politischen Kommunikation und diskutiert verschiedene Ansätze, die Privatisierung als rationale Strategie begreifen. Der Blick auf internationale Studien bietet Ansatzpunkte, die Tina Rohowski für ihre eigene Untersuchung handhabbar macht. Ihr Fazit zum Forschungsstand: Vor allem Langzeitstudien zum Phänomen der Privatisierung in der deutschen Presse existierten nicht, eine Zunahme der Privatheit sei empirisch bislang nicht belegt.

Die Autorin setzt mit ihrer empirischen Untersuchung nahtlos an den von ihr herausgearbeiteten Desideraten an. Sie leitet zwei zentrale Hypothesen ab: 1) Deutsche Medien berichten in immer größerem Umfang über das Privatleben der Politiker. 2) Die Berichte selbst werden zunehmend persönlicher; sie stellen also immer größere Teile des Privatlebens "hinter“ dem Amtsträger aus. Es geht also sowohl darum, die Quantität der Homestories zu analysieren, als auch ihre Qualität.

Im Rahmen einer Inhaltsanalyse untersucht die Autorin die Illustrierten Bunte und Stern seit ihrer Gründung 1948 bis zum Jahr 2007. Die Auswahl scheint plausibel, allerdings muss bezweifelt werden, dass sich allein aus der Analyse der Berichterstattung zweier Illustrierter, die sich ohnehin der sog. „People-Berichterstattung" verschrieben haben, ein allgemeiner Trend zur Personalisierung ableiten lässt, wie die Autorin postuliert (problematisch S. 76). Vielmehr muss die Aussagekraft zwangsläufig begrenzt bleiben. Man könnte entgegenhalten, dass sich eine Privatisierung eben nicht in den Illustrierten, sondern vielmehr in den sog. Qualitätsmedien zeigt, die entgegen ihrem vermeintlichen Anspruch - Politikberichterstattung - zunehmend auch Politikerberichterstattung leisten.

Für die Analyse der insgesamt 169 Homestories erarbeitet die Autorin in Anlehnung an Ana Inés Langer einen Kriterienkatalog, der insgesamt 19 Themen (etwa Kindheit \& Jugend, Freundeskreis, Finanzen etc.) umfasst und den Kern der inhaltlichen Kodierung bildet. Allerdings kann man hinterfragen, ob die Zahl der angesprochenen Themen tatsächlich ein valides Mittel ist, um das Maß an Privatheit zu analysieren. Denkbar ist auch, dass ein monothematischer Artikel eine viel größere Nähe zum dargestellten Politiker bietet als wenige kurze Statements zu verschiedenen Themen.

Tina Rohowski kommt bei der Analyse der in den Artikeln behandelten Themen zu dem Schluss: „Die Politiker machen die Tür zu ih- rem Privatleben nicht nur immer häufiger, sondern auch immer weiter auf“" (S. 85). Die Stärke der Arbeit liegt in den Ergebnissen, die Tina Rohowski aus der umfangreichen Langzeitanalyse gewinnt, wie zum Beispiel die Tatsache, dass einige Themen zu den "Dauerbrennern“ der Privatisierung gehören - so die Einbeziehung des (Ehe-)Partners. Es scheint Muster zu geben, die - unabhängig vom gesellschaftlichen Wandel - den Akteuren in den frühen sechziger Jahren genauso gewinnbringend erschienen wie den Politikern von heute. Bemerkenswert ist auch die Erkenntnis der Autorin, dass die Zahl der Homestories, die sich zusätzlich mit politischen Themen befasst, zugenommen hat: Die Berichte werden privater und zugleich politischer. In der qualitativen Auswertung der Ergebnisse beschreibt Tina Rohowski detailliert, welche sprachlichen Muster die Homestories aufweisen.

Die Arbeit füllt erfolgreich die Forschunglücke, die die Autorin zu Beginn aufzeigt. Die Befunde zur Genese der Homestories sind für das Fach hilfreich und können dazu beitragen, eine normativ aufgeheizte Debatte zu versachlichen. Allerdings ist eine weiter reichende theoretische Verortung des Themas wünschenswert: So lässt die Autorin offen, wen sie als Urheber der Privatisierung versteht - die Journalisten oder gar die Politiker? Hier spricht sie nur vage von „Kooperation“ (S. 60). Auch stellt sich dem Leser die Frage nach den Gründen für eine solche Privatisierung: Warum lässt Scharping sich im Pool interviewen? Warum spricht Kinkel über das Haarefärben? Diese Fragen - ebenso wie den von der Autorin aufgeworfenen Aspekt der Medienwirkungen müssen aufbauende Arbeiten beantworten.

Barbara Hans

\section{Pelle Snickars / Patrick Vonderau (Hrsg.) The YouTube Reader}

Stockholm: National Library of Sweden, 2009. $-512 \mathrm{~S}$.

ISBN 978-9-188468-11-6

Vor 1990 hätten Prognosen eines Bewegtbildangebots, das den Programmumfang des Fernsehens übersteigt, ein mitleidiges Lächeln von Fernsehwissenschaftlern ausgelöst. Jetzt zählen täglich neu hochgeladene Videos zum Alltag vieler Produser. Die beiden Medienwissenschaftler Pelle Snickars und Patrick Vonderau verweisen auf die schnelle Entwicklung YouTubes von einer Videotauschbörse hin zu der Video Community im Netz (S. 10): Grund 